Working Paper 98-96

Statistics and Econometrics Series 42

December 1998
Departamento de Estadística y Econometría

Universidad Carlos III de Madrid

Calle Madrid, 126

28903 Getafe (Spain)

Fax 34 - 91- 624.9849

OUTLIERS IN MULTIVARIATE TIME SERIES

Ruey S. Tsay, Daniel Peña and Alan E. Pankratz*

\begin{abstract}
This paper considers outliers in multivariate time series analysis. It generalizes four types of disturbances commonly used in the univariate time series analysis to the multivariate case, and investigates dynamic effects of a multivariate outlier on individual components if marginal models are used. An innovational outlier of a vector series can introduce a patch of outliers for the marginal component models. The paper also proposes an iterative procedure to detect and handle multiple outliers. By comparing and contrasting results of univariate and multivariate outlier detections, one can gain insights into the characteristics of an outlier. An outlier in a component series may or may not have significant impacts on the other components. We use real examples to demonstrate the proposed analysis.
\end{abstract}

Key Words

Additive outlier; innovational outlier; level shift; temporary change.

"Tsay, Graduate School of Business, University of Chicago, USA; Peña, Department of Statistics and Econometrics, Universidad Carlos III de Madrid, e-mail: dpena@est-econ.uc3m.es; Pankratz, Department of Economics \& Management, DePawn University, USA. 


\title{
Outliers in Multivariate Time Series
}

\author{
Ruey S. Tsay, Graduate School of Business, University of Chicago \\ Chicago, IL 60637, USA \\ Daniel Peña, Department of Statistics \& Econometrics \\ Universidad Carlos III, Madrid, Spain \\ Alan E. Pankratz, Department of Economics \& Management \\ DePauw University, Greencastle, IN 46135, USA
}

\begin{abstract}
This paper considers outliers in multivariate time series analysis. It generalizes four types of disturbances commonly used in the univariate time series analysis to the multivariate case, and investigates dynamic effects of a multivariate outlier on individual components if marginal models are used. An innovational outlier of a vector series can introduce a patch of outliers for the marginal component models. The paper also proposes an iterative procedure to detect and handle multiple outliers. By comparing and contrasting results of univariate and multivariaie outlier detections, one can gain insights into the characteristics of an outlier. An outlier in a component series may or may not have significant impacts on the other components. We use real examples to demonstrate the proposed analysis.
\end{abstract}

Keywords: Additive outlier, Innovational outlier, Level shift, Temporary change.

\section{Introduction}

Outlier detection has become an integral part of statistical data analysis. In the time series literature, outlier detection plays an important role in modeling, inference, and even data processing because outliers can lead to model misspecification, biased parameter estimation, and poor forecasts. As a specific example, outlier detection has become a key feature in recent advance in seasonal adjustment and in automatic time-series model identification; see the new adjustment procedure X-12 ARIMA of Findley, et al (1998), which is used by the U.S. Government, and the SEATS and TRAMO programs of Gomez and Maravall (1994a, b), which was recently adopted by the European Union. Following the work of Fox (1972), four types of outlier have been proposed for univariate time series analysis and several methods are available to detect these outliers. The outliers are classified as (a) additive outlier (AO), (b) innovational outlier (IO), (c) level shift (LS), and temporary change (TC). These four types of outlier affect an observed time series and its residual process differently. For example, an $\mathrm{AO}$ affects a single observation, but several residuals, depending on the underlying model. See Chang, Tiao and Chen (1988), Chen and Liu (1993), and Tsay (1988) and the references therein. For outlier detection, both Bayesian and non-Bayesian methods have been proposed. See Justel, Peña and Tsay (1998) and the references therein for Bayesian methods and Chen and Liu (1993) and the references therein for non-Bayesian methods.

However, most outlier studies in time series analysis focus on a single series. A commonpractice for handling outliers in a multivariate process is to apply univariate techniques to the component series 
to remove outlier effects, then treat the adjusted series as outlier free and model them jointly. This procedure encounters several difficulties. First, in a multivariate process, an outlier of a component may be due to an outlier in the other components. Overlooking such a possibility can easily lead to overspecification of the number of outliers. Second, an outlier of moderate size affecting all the components maybe unnoticed in the univariate analyses because univariate methods fail to combine information about the outlier among the component series. This outlier will be more easily detected in multivariate analysis. Third, univariate detection procedures often use inferior estimation, because the joint dynamics of the series are not properly taken into account.

Pankratz (1993) considers AO and IO in a dynamic regression model with a single input and a single output. He classifies outliers in the input series as passed and non-passed outliers and uses a weighted average of least squares estimators to estimate non-passed outliers. The approach becomes complicated when there are multiple input or multiple output series.

The main objective of this paper is to study outliers directly under a multivariate framework. As such, the proposed method of outlier detection can overcome the aforementioned difficulties. Furthermore, by comparing and contrasting results of univariate and multivariate detection methods, one can gain insight into the characteristics of an outlier. For example, consider a transfer function model consisting of a single input series and a single output series. Assume that the delay from the input series to the output series is one time period. Then, a single innovational outlier of the input series at time $t$ will show its effect on the output series at time $t+1$. In this case, if one applies a univariate outlier detection method separately to the two component series, one would identify an IO for the input series at time $t$ and another outlier for the output series at time $t+1$. On the other hand, if one uses the proposed multivariate detection method, he would only identify an IO at time $t$, because the effects of the outlier on the output series at time $t+1$ are taken care of automatically under a multivariate framework. We shall demonstrate this situation later by a real example.

The paper is organized as follows. We generalize the four types of outlier to the multivariate case in Section 2. In Section 3, we consider estimation of outlier parameters, assuming that the time series model is known. Two test statistics are considered for each type of outliers using the estimates of outlier parameters. The first test statistic is a joint statistic that combines information across components and the second test statistic is marginal and uses information contained in an individual component. An iterative procedure is then proposed in Section 4 for practical outlier detection. Section 5 contains two real examples.

\section{Outliers in a Vector Time Series}

Let $x_{t}=\left(x_{1 t}, \cdots, x_{k t}\right)^{\prime}$ be a $k$-dimensional time series that follows a vector autoregressive integrated moving-average (ARIMA) model

$$
\Phi(B) x_{t}=c+\Theta(B) \epsilon_{t}
$$

where $\Phi(B)=I-\Phi_{1} B-\cdots-\Phi_{p} B^{p}$ and $\Theta(B)=I-\Theta_{1} B-\cdots-\Theta_{q} B^{q}$ are $k \times k$ matrix polynomials of finite degrees $p$ and $q, B$ is the backshift operator such that $B x_{t}=\mathbf{x}_{t-1}, c$ is a $k$-dimensional constant vector, and $\left\{\epsilon_{t}=\left(\epsilon_{1 t}, \cdots, \epsilon_{k t}\right)^{\prime}\right\}$ is a sequence of independent and identically distributed Gaussian random vectors with mean zero and positive-definite covariance matrix $\boldsymbol{\Sigma}$. We assume that $\Phi(B)$ and $\Theta(B)$ are left coprime and all of the zeros of the determinants $|\Phi(B)|$ and $|\Theta(B)|$ are on or outside the unit circle. In addition, if $|\Phi(1)|=0$, we assume that the series $x_{t}$ starts at a fixed time point $t_{0}$ with fixed initial values and initial innovations. The series $x_{t}$ is (asymptotically) stationary if $|\Phi(1)| \neq 0$ and is unit-root nonstationary, otherwise. Similarly, $x_{t}$ is invertible if $|\Theta(1)| \neq 0$ and 
is non-invertible, otherwise. See $\mathrm{Li}$ and Tsay (1998) for further information.

For the vector ARIMA model in (1), define the autoregressive representation as

$$
\Pi(B) x_{t}=c_{0}+\epsilon_{t}
$$

where $c_{0}=[\Theta(1)]^{-1} c$ if $x_{t}$ is invertible and it is a function of $t, c, \Theta_{i}$, initial values and initial innovations if $x_{t}$ is non-invertible, $\Pi(B)=I-\sum_{i=1}^{\infty} \Pi_{i} B^{i}=[\Theta(B)]^{-1} \Phi(B)$ and it is understood that $y_{t}=0$ if $t<t_{0}$, and the moving-average representation as

$$
x_{t}=c_{*}+\Psi(B) \epsilon_{t}
$$

where $\Psi(B)=I+\sum_{i=1}^{\infty} \Psi_{i} \epsilon_{t-i}=[\Theta(B)]^{-1} \Phi(B)$, and $c_{*}=[\boldsymbol{\Phi}(1)]^{-1} c=E\left(x_{t}\right)$ if $\boldsymbol{x}_{\boldsymbol{t}}$ is stationary and it is a function of $t, c, \Phi_{i}$, initial values and initial innovations if $x_{t}$ is unit-root nonstationary. Obviously, we have $[\Pi(B)]^{-1} \Psi(B)=[\Psi(B)]^{-1} \Pi(B)=I, \Pi(1) c_{*}=c_{0}$ and $\Psi(1) c_{0}=c_{*}$.

Let $\xi_{t}^{(h)}$ be the indicator variable for time index $h$, i.e. $\xi_{h}^{(h)}=1$ and $\xi_{t}^{(h)}=0$ if $t \neq h$. Denote the observed time series by $\boldsymbol{y}_{t}=\left(y_{1 t}, \cdots, y_{k t}\right)^{\prime}$, and let $\boldsymbol{\omega}=\left(\omega_{1}, \cdots, \omega_{k}\right)^{\prime}$ be the size of the initial impact of an outlier on the series $\boldsymbol{x}_{t}$. Following the univariate case, we consider four types of outlier for $\boldsymbol{x}_{t}$.

1. Innovational outlier (IO): An IO represents an unexpected change in the innovations that drive the vector time series. For instance, suppose that the noise in a bivariate series consisting of oven temperature and a chemical concentration reading is mainly due to the random variability of the feed rate. Then a sudden change in the feed rate that happens at just a particular time point, due to some exogenous effect, will produce an $1 O$ in the series. The model for an IO at time $h$ is

$$
y_{t}=x_{t}+\Psi(B) \omega \xi_{t}^{(h)}=c_{*}+\Psi(B)\left(\epsilon_{t}+\omega \xi_{t}^{(h)}\right)
$$

2. Additive outlier (AO): An $A O$ represents an unexpected change in one of the observations. It can appear because of a recording or measurement error or other single effect. For instance, a short strike in a production process may introduce an $A O$ in a multivariate output series. The model for an AO at time $h$ is

$$
y_{t}=\boldsymbol{\omega} \xi_{t}^{(h)}+x_{t} .
$$

3. Level shift (LS): A LS represents an unexpected shift in all the values of the observed time series after some time point. Level shifts affecting all the components of a vector time series are sometimes called structural breaks, because they produce a permanent effect on the vector series. For instance, a currency devaluation may affect exports and imports of a country. A policy change or a change in definition may produce a permanent shifts in quarterly unemployment rate and claims of unemployment benefits. The model for a LS at time $h$ is

$$
y_{t}=x_{t}+\omega \frac{1}{1-B} \xi_{t}^{(h)}
$$

4. Temporary change (TC): A TC represents an unexpected change on the values of a time series that disappears after a short period of time. For instance, the effect of a price promotion on sales of a product may disappear over time. The model for a temporary change at time $h$ is

$$
y_{t}=x_{t}+\omega \frac{1}{1-\delta B} \xi_{t}^{(h)}
$$


where $\boldsymbol{\omega}$ is the initial change in the level and this change decays exponentially over time at the rate $\delta$, where $0<\delta<1$. Notice that if $\delta$ is close to zero then a TC is very similar to an AO, whereas if $\delta$ is close to unity, then a TC is similar to a LS. Consequently, to avoid ambiguity in outlier classification, we use $\delta=0.7$ in this paper, which allows approximately ten periods of decreasing effects because $0.7^{10} \approx .028$.

Note that if some components of $\boldsymbol{\omega}$ are zero, then the outlier does not occur in the corresponding component of $y_{t}$. Also, in some applications $\boldsymbol{\omega}$ may have restrictions. For instance, suppose that the components of the vector time series represent market shares. Then, an increase in level of one component is equal to the total decrease in level of other components. Here the linear constraint $\sum_{i} \omega_{i}=0$ applies, where $\omega_{i}$ is the $i$-th element of $\omega$. This type of restrictions can be incorporated easily into the multivariate framework, even though we do not specifically discuss it in this paper.

In practice, disturbances to a vector time series may not follow one of the four categories discussed. But as shown by the univariate analysis, these four types of outlier and their linear combinations are capable of providing good approximations to describe most disturbances commonly encountered in time-series applications.

\subsection{Implications to marginal models}

From the definitions, an AO, LS, or TC in a multivariate time series implies an AO, LS, or TC for the marginal models of individual components. However, an IO in a multivariate model may introduce a patch of outliers for the marginal models. To see this, let us consider the simplest case of a bivariate MA(1) model with an IO at time index $h$ :

$$
\left[\begin{array}{l}
y_{1 t} \\
y_{2 t}
\end{array}\right]=\left[\begin{array}{rr}
1-\Theta_{11} B, & -\Theta_{12} B \\
-\Theta_{21} B, & 1-\Theta_{22} B
\end{array}\right]\left(\left[\begin{array}{l}
\epsilon_{1 t} \\
\epsilon_{2 t}
\end{array}\right]+\left[\begin{array}{l}
\omega_{1} \\
\omega_{2}
\end{array}\right] \xi_{t}^{(h)}\right) .
$$

For this particular instance, the marginal model for $x_{1 t}$ is a univariate MA(1) model, say $x_{1 t}=$ $(1-\theta B) e_{1 t}$, where $\left\{e_{1 t}\right\}$ is a white noise sequence with mean zero and variance $\sigma_{e}^{2}$ and the parameters $\theta$ and $\sigma_{e}^{2}$ are determined by the relationships

$$
\left(1+\Theta_{11}^{2}\right) \sigma_{11}+\Theta_{12}^{2} \sigma_{22}+2 \Theta_{11} \Theta_{12} \sigma_{12}=\left(1+\theta^{2}\right) \sigma_{e}^{2}, \quad-\Theta_{11} \sigma_{11}-\Theta_{12} \sigma_{12}=-\theta \sigma_{e}^{2},
$$

where $\sigma_{i j}$ is the $(i, j)$-th element of $\operatorname{cov}\left(\epsilon_{t}\right)$. The marginal model for the observed series $y_{1 t}$ is then

$$
y_{1 t}=(1-\theta B) e_{1 t}+\omega_{1} \xi_{t}^{(h)}-\left(\Theta_{11} \omega_{1}+\Theta_{12} \omega_{2}\right) \xi_{t-1}^{(h)}
$$

Because the quantity $\omega_{2}$ in the last term of the right side of equation (5) does not appear in the second term, we can choose $\omega_{2}$ so that the outlier effect on $y_{1 t}$ cannot be written as $(1-\theta B) \omega_{1} \xi_{t}^{(h)}$. This shows that the outlier at the time index $h$ is no longer a simple IO for $y_{1 t}$. Consequently, we have two consecutive outliers at time indexes $h$ and $h+1$ for the marginal model of $y_{1 t}$. This result can easily be extended to the general vector ARIMA models. It says that an IO of a multivariate model may introduce a patch of outliers in the marginal models of the components. The length of the outlier patch depends on the order of the model for $x_{t}$. This result can help explain the empirical finding that univariate outlier detection often identifies consecutive outliers. See Example 1 of Section 5. 


\subsection{Effects of outliers}

Similar to the univariate case, an outlier can introduce biases in parameter estimation and misspecification in model identification. It pays to understand the effects of an outlier on the observed series and on residuals. In fact, most outlier detection methods make use of the behavior of residuals implied by an outlier. Assume that the model of $x_{t}$ is known. Define a filtered series $\left\{a_{t}\right\}$ by

$$
a_{t}=y_{t}-\sum_{i=1}^{p} \Phi_{i} y_{t-i}-c+\sum_{j=1}^{q} \Theta_{j} a_{t-j}, \quad t=t_{0}, t_{0}+1, \cdots
$$

where $y_{t}=x_{t}$ and $a_{t}=\epsilon_{t}$ for $t<t_{0}$. That is, $y_{t}$ and $x_{t}$ have the same initial values and initial innovations. In terms of $\mathrm{AR}$ representation, we have $\Pi(B) y_{t}=c_{0}+a_{t}$. By definition, if there exist no outliers, then $a_{t}=\epsilon_{t}$. In the presence of outliers, $a_{t} \neq \epsilon_{t}$ for some time points. The number of $a_{t}$ affected by an outlier depends on the dynamic of $x_{t}$ as well as the type of the outlier. Details are given below.

1. IO case: Applying $\Pi(B)$ to the series $y_{t}$, we have

$$
a_{t}=\omega \xi_{t}^{(h)}+\epsilon_{t}
$$

Therefore, an IO only affects a single filtered value at the time of occurrence.

2. AO case: Multiplying $\Pi(B)$ from left to the model and subtracting $c_{o}$ from both sides of the equation, we have

$$
\boldsymbol{a}_{t}=\Pi(B) \boldsymbol{\omega} \xi_{t}^{(h)}+\boldsymbol{\epsilon}_{\boldsymbol{t}} .
$$

Consequently, when $\Pi(B) \neq I$, an AO at time $h$ affects multiple filtered values of $a_{t}$ at $t=$ $h, h+1, \cdots$. For a vector $\mathrm{AR}(p)$ model, it will affect $\boldsymbol{a}_{t}$ for $t=h, h+1, \cdots, h+p$.

3. LS case: Using the same technique as the $A O$ case, we have

$$
a_{t}=\Pi^{*}(B) \omega \xi_{t}^{(h)}+\epsilon_{t}
$$

where $\Pi^{*}(B)=\Pi(B) /(1-B)$. Here it is clear that a LS at time $h$ affects all filtered values $a_{t}$ for $t \geq h$.

4. TC case: Similarly, we have

$$
a_{t}=\Pi^{\circ}(B) \omega \xi_{t}^{(h)}+\epsilon_{t}
$$

where $\Pi^{o}(B)=\Pi(B) /(1-\delta B)$. Because $\delta<1$, it is seen that a TC at time $h$ affects all $\boldsymbol{a}_{t}$ for $t \geq h$, but the effects decay exponentially as $t-h$ increases.

The above results are generalizations of those of the univariate case. However, what is buried under the formulas is the outlier impact induced by the dynamic relation among the components of $x_{t}$. An outlier of the component $y_{i t}$ at time index $h$ may or may not affect the filtered components $a_{j t}$ for $j \neq i$ and $t \geq h$. Furthermore, when there is cross-dependence among components of $x_{t}, a_{i t}$ of the filtered series contains information about outliers of component $y_{j t}$. Consequently, a vector series when considered jointly contains more information about an outlier than does a univariate series. Consider the case of a bivariate system $\left(x_{1 t}, x_{2 t}\right)$ in which $x_{1 t}$ is the input and $x_{2 t}$ the output. Suppose that an AO is detected at time $h$ in the univariate analysis of the input series. We cannot 
tell from the analysis whether the outlier was due to (a) a recording or measurement error or (b) an intervention that really changed the value of the series at time index $h$. However, if the multivariate analysis also shows significant outlier effects in the output series at the same time index, then it is more likely that the $\mathrm{AO}$ is due to an intervention that affects both series. On the other hand, if the multivariate analysis fails to show any significant outlier effects in the output series at time index $h$, then the chance of a recording error increases because the analysis shows that the output series is consistent with the outlier-adjusted input series. See Example 2 of Section 5 for an illustration.

\section{Outlier Estimation and Testing}

The filtered series $\boldsymbol{a}_{t}$ of the previous subsection provides a natural way to estimate outlier parameter $\boldsymbol{\omega}$. Assuming that model (1) of $x_{t}$ is known so that $a_{t}$ is available, we shall use multiple linear regression to estimate the size $\boldsymbol{\omega}$ of a single outlier. In practice, the parameters of model (1) need to be estimated and $a_{t}$ becomes the residuals of a fitted model. We then use an iterative procedure in outlier detection to overcome the difficulty of assuming that $\Pi(B)$ and $\boldsymbol{\Sigma}$ are known.

Consider the case of an IO at time index $h$. All information about the outlier is contained in $a_{h}$. Therefore, we estimate the outlier using $\hat{\omega}_{I, h}=a_{h}$, where the subscript $I$ indicates IO, and derive test statistics based on the estimate. Two test statistics are considered. The first one is a joint test that treats $\hat{\omega}_{I, h}$ as a multivariate quantity and the other test is based on component statistics that treats each component of $\hat{\boldsymbol{\omega}}_{I, h}$ separately. These two test statistics represent the two extreme cases in making use of the available information. The basic framework for outlier detection is to consider the null hypothesis $H_{0}: \boldsymbol{\omega}=\mathbf{0}$ versus the alternative hypothesis $H_{a}: \boldsymbol{\omega} \neq \mathbf{0}$ for a given time index $h$. For the joint test that considers all elements of $\boldsymbol{\omega}$ simultaneously, we use the test statistic

$$
J_{I, h}=a_{h}^{\prime} \Sigma^{-1} a_{h}
$$

where $J$ stands for a "joint statistic." Under the assumption of knowing the model in (1) and the null hypothesis of no IO, $J_{I, h}$ has a chi-square distribution with $k$ degrees of freedom for a given $h$.

The second statistic is based on the belief that an IO may occur only in some component of $\boldsymbol{x}_{t}$. In this case, the joint statistic in (10) may not be powerful in detecting outliers, and we use the test statistic

$$
C_{I, h}=\max _{1 \leq i \leq k}\left|a_{i, h}\right| / \sqrt{\sigma_{i i}}
$$

where "C" stands for "component statistic," $a_{i, h}$ is the $i$-th component of $a_{h}$ and $\sigma_{i i}$ is the $(i, i)$-th element of the $\boldsymbol{\Sigma}$ matrix. This test statistic is the maximum of individual $t$-ratios, in absolute value, of the components of $a_{h}$.

For the other types of outlier, a multiple linear regression is needed. Let $\Sigma^{1 / 2}$ be the square root of the covariance matrix $\Sigma$. Define $e_{t}=\Sigma^{-1 / 2} \epsilon_{t}$. Then, $e_{t}$ is a sequence of independent and identically distributed Gaussian random vectors with mean zero and covariance matrix $I$, the identify matrix. Because the same idea applies to AO, LS, and TC, we shall give details for the AO case only. Multiplying equation (7) from left by $\Sigma^{-1 / 2}$ and noticing that the AO at time index $h$ only affects $a_{t}$ for $t \geq h$, we have

$$
b_{t}=\Lambda(B) \omega \xi_{t}^{(h)}+e_{t}, \quad t=h, h+1, \cdots, n
$$

where $b_{t}=\boldsymbol{\Sigma}^{-1 / 2} a_{t}$ and $\Lambda(B)=\Lambda_{0}-\sum_{j=1}^{\infty} \Lambda_{j} B^{j}=\boldsymbol{\Sigma}^{-1 / 2} \boldsymbol{\Pi}(B)$. It is understood that in (12) $t$ is from $h$ to $h+p$ if $x_{t}$ follows a pure AR model. Because $e_{t}$ has no serial correlations and $\operatorname{cov}\left(e_{t}\right)=$ 
$I$, the univariate sequence $\left\{e_{1, h}, \cdots, e_{k, h}, e_{1, h+1}, \cdots, e_{k, h+1}, e_{1, h+2}, \cdots\right\}$ is a sequence of independent and identically distributed standard Gaussian random variates. Consequently, equation (12) can be used to form a multiple linear regression for the unknown parameter $\boldsymbol{\omega}$. The regressors are columns of the coefficient matrices of $\Lambda(B)$, the dependent variable consists of elements of $\boldsymbol{b}_{t}$. Each equation in (12) contributes $k$ data points so that the number of data points for the multiple linear regression is $k(n-h+1)$, where $k$ is the dimension of $x_{t}$. More specifically, we have

$$
\begin{aligned}
\boldsymbol{b}_{h} & =\boldsymbol{\Lambda}_{0} \boldsymbol{\omega}+e_{h} \\
\boldsymbol{b}_{h+1} & =-\boldsymbol{\Lambda}_{1} \boldsymbol{\omega}+\boldsymbol{e}_{h+1} \\
\vdots & =\vdots
\end{aligned}
$$

where each equation contributes $k$ data points to the multiple linear regression. Denote the ordinary least squares estimate of $\boldsymbol{\omega}$ by $\hat{\boldsymbol{\omega}}_{A, h}$, where the subscript $A$ indicates additive outlier. The covariance matrix of $\hat{\omega}_{A, h}$ is simply the usual $X^{\prime} X$-matrix because the variance of the error term of the multiple linear regression is unity. For ease in reference, we denote the covariance matrix of $\hat{\boldsymbol{\omega}}_{A, h}$ by $\boldsymbol{\Sigma}_{A, h}$.

To test the significance of the AO at time index $h$, we consider the null hypothesis $H_{0}: \boldsymbol{\omega}=\mathbf{0}$ versus the alternative hypothesis $H_{a}: \boldsymbol{\omega} \neq 0$. Again, two test statistics are used. The first test statistic is

$$
J_{A, h}=\hat{\omega}_{A, h}^{\prime} \Sigma_{A, h}^{-1} \hat{\omega}_{A, h},
$$

that treats components of $\boldsymbol{\omega}$ jointly. For a fixed $h$ and assuming that the model is known, $J_{A, h}$ is distributed as a chi-square random variable with $k$ degrees of freedom under the null hypothesis. The second test statistic used is the maximum $t$-ratio (in absolute value) of components of $\hat{\omega}_{A, h}$. That is,

$$
C_{A, h}=\max _{1 \leq i \leq k}\left|\hat{\omega}_{i, A, h}\right| / \sqrt{\sigma_{i, A, h}}
$$

where $\hat{\omega}_{i, A, h}$ and $\sigma_{i, A, h}$ are the $i$-th element of $\hat{\boldsymbol{\omega}}_{A, h}$ and the $(i, i)$-th element of $\boldsymbol{\Sigma}_{A, h}$, respectively.

Define $\Lambda^{*}(B)=\Lambda_{0}^{*}-\sum_{j=1}^{\infty} \Lambda_{j}^{*} B^{j}=\Sigma^{-1 / 2} \Pi^{*}(B)$. We can apply the same techniques as those of the AO case to obtain an estimate of the size of LS at time index $h$. Denote the estimate and its covariance matrix by $\hat{\boldsymbol{\omega}}_{L, h}$ and $\boldsymbol{\Sigma}_{L, h}$, respectively. The significance of this estimate can then be tested using the joint statistic

$$
J_{L, h}=\hat{\boldsymbol{\omega}}_{L, h}^{\prime} \Sigma_{L, h}^{-1} \hat{\boldsymbol{\omega}}_{L, h}
$$

which, again, under the null hypothesis of no level shift, follows a chi-square distribution with $k$ degrees of freedom. The second test statistic for LS is

$$
C_{L . h}=\max _{1 \leq i \leq k}\left|\hat{\omega}_{i, L, h}\right| / \sqrt{\sigma_{i, L, h}}
$$

where $\hat{\omega}_{i, L, h}$ and $\sigma_{i, L, h}$ are defined in a similar manner as $\hat{\omega}_{i, A, h}$ and $\sigma_{i, A, h}$ in equation (14).

Finally, define $\Lambda^{o}(B)=\Lambda_{0}-\sum_{j=1}^{\infty} \Lambda_{j}^{o} B^{j}=\Sigma^{-1 / 2} \Pi^{o}(B)$. We can obtain an estimate of the size of a temporary change at time $h$ and its covariance matrix. Denote them as $\hat{\boldsymbol{\omega}}_{T, h}$ and $\boldsymbol{\Sigma}_{T, h}$, respectively. The significance of this TC can be checked by using the joint test statistic

$$
J_{T, h}=\dot{\omega}_{T, h}^{\prime} \Sigma_{T, h}^{-1} \hat{\boldsymbol{\omega}}_{T, h}
$$

which, under proper assumptions, follows a chi-square distribution with $k$ degrees of freedom. The second test statistic for $\mathrm{TC}$ is

$$
C_{T . h}=\max _{1 \leq i \leq k}\left|\hat{\omega}_{i, T, h}\right| / \sqrt{\sigma_{i, T, h}}
$$

where $\hat{\omega}_{i, T, h}$ and $\sigma_{i, T, h}$ are the $i$-th component of $\hat{\boldsymbol{\omega}}_{T, h}$ and the $(i, i)$-th element of $\boldsymbol{\Sigma}_{T, h}$, respectively. 


\subsection{Outlier detection when time index is unknown}

When the time index $h$ of an outlier is unknown, we check all observations to detect outliers. To this end, we make use of the statistics in (10) to (18) and define the overall test statistics

$$
\begin{aligned}
& J_{\max }\left(I, h_{I}\right)=\max _{h} J_{I, h}, \quad C_{\max }\left(I, h_{I}^{*}\right)=\max _{h} C_{I, h} \\
& J_{\max }\left(A, h_{A}\right)=\max _{h} J_{A, h}, \quad C_{\max }\left(A, h_{A}^{*}\right)=\max _{h} C_{A, h} \\
& J_{\text {max }}\left(L, h_{L}\right)=\max _{h} J_{L, h}, \quad C_{\max }\left(L, h_{L}^{*}\right)=\max _{h} C_{L, h} \\
& J_{\text {max }}\left(T, h_{T}\right)=\max _{h} J_{T, h}, \quad C_{\max }\left(T, h_{T}^{*}\right)=\max _{h} C_{T, h}
\end{aligned}
$$

where it is understood that $h_{i}$ denotes the time index when the maximum of test statistic $J_{i, h}$ occurs and $h_{i}^{*}$ denotes the time index when the maximum of $C_{i, h}$ occurs, where $i=I, A, L, T$. Under the null hypothesis of no outlier in the sample and assuming that the model of $x_{t}$ is known, $J_{\max }\left(I, h_{I}\right)$ is the maximum of a random sample of size $n$ from a chi-square distribution with $k$ degrees of freedom. Thus, the asymptotic distribution of $J_{\max }\left(I, h_{I}\right)$ can be obtained using the extreme value distribution. Each of the other three joint test statistics in (19) is the maximum of a dependent sample from a chi-square distribution with $k$ degrees of freedom. Their asymptotic distributions are therefore more complicated, depending on the serial dependence of $\left\{J_{i, h}\right\}$. From the estimation of the outlier parameter $\omega$, it is seen that the serial correlations of $\left\{J_{L, h}\right\}_{h=1}^{n}$ are stronger than those of $\left\{J_{i, h}\right\}$ for $i=I, A, T$. This is due to the nondecaying weights induced by the operator $1 /(1-B)$ so that $\hat{\boldsymbol{\omega}}_{L, h}$ contains all of the filtered values $a_{t}$ for $t \geq h$. Consequently, the asymptotic distribution of $J_{\max }\left(L, h_{L}\right)$ is more concentrated than those of the other three joint test statistics. Thus, the critical values of $J_{\max }\left(L, h_{L}\right)$ are in general smaller than those of the other joint test statistics. In sum, $\left\{J_{i, h}\right\}$ are sequences of chi-square samples with $k$ degrees of freedom, where $i=I, A, L, T$. The sequence $\left\{J_{I, h}\right\}$ is serially uncorrelated whereas the sequence $\left\{J_{L, h}\right\}$ has the strongest serial correlations. The critical values of $J_{\max }\left(i, h_{i}\right)$ for $i=A$ and $T$ should be between those of $J_{\max }\left(I, h_{I}\right)$ and $J_{\max }\left(L, h_{L}\right)$.

For the component test statistics $C_{\max }\left(i, h_{i}^{*}\right)$, the critical values should be close to those commonly used in the univariate outlier detection, because these statistics are based on individual components. The only difference in the multivariate case is that the maximization is evaluated across the $k$ components as well as over the time indices. Similar to the joint test statistics, asymptotic distributions of $C_{\max }\left(i, h_{i}^{*}\right)$ also depend on the serial correlations of $\left\{C_{i, h}\right\}$. In this paper, we use simulation to generate finite sample critical values of these test statistics.

\subsection{Simulation}

We employ two vector $A R(1)$ models in our simulation to obtain empirical quantiles of the test statistics in (19) for $k=2,3$ and for sample sizes $n=100,200$, and 400 . The two models are in the form $x_{t}=\boldsymbol{\Phi} x_{t-1}+\epsilon_{t}$ with parameters

$$
\boldsymbol{\Phi}=\left[\begin{array}{rr}
0.2 & 0.3 \\
-0.6 & 1.1
\end{array}\right], \quad \boldsymbol{\Sigma}=\left[\begin{array}{ll}
1.0 & 0.2 \\
0.2 & 1.0
\end{array}\right]
$$

for the bivariate case and

$$
\boldsymbol{\Phi}=\left[\begin{array}{rrr}
0.2 & 0.3 & 0.0 \\
-0.6 & 1.1 & 0.0 \\
0.2 & 0.3 & 0.6
\end{array}\right], \quad \boldsymbol{\Sigma}=\left[\begin{array}{lll}
1.0 & 0.2 & 0.2 \\
0.2 & 1.0 & 0.2 \\
0.2 & 0.2 & 1.0
\end{array}\right]
$$


for the trivariate case. Because of the normalization by the matrix $\boldsymbol{\Sigma}^{1 / 2}$, the proposed detection statistics in (19) are scale-invariant. Therefore, the two models used in our simulation represent general vector $A R(1)$ models whose coefficient matrix $\Phi$ has eigenvalue $(0.5,0.8)$ and $(0.5,0.6,0.8)$, respectively.

For a given model and sample size $n$, we generate 10,000 realizations. For each realization, we estimate a VAR(1) model by the ordinary least squares method, obtain the residuals and $\hat{\boldsymbol{\Sigma}}$, and compute the test statistics in (19) using the estimated parameters. Tables 1 and 2 provide some empirical quantiles of the test statistics under the null hypothesis of no outliers in the data. Table 1 is for the joint statistics whereas Table 2 is for the component statistics. From the tables, we make the following observations. First, as expected, empirical quantiles of $J_{\max }\left(L, h_{L}\right)$ are much smaller than those of the other three joint test statistics. Second, quantiles of $J_{\max }\left(i, h_{i}\right)$ for $i=I, A, T$ are close to each other, implying that a common critical value can be used for these three test statistics. Third, empirical quantiles of the component statistics $C_{\max }\left(i, h_{i}^{*}\right)$ are more variable when $k=3$. The quantiles of $C_{\max }\left(L, h_{L}^{*}\right)$ are smaller than those of the other component statistics for $k=2$. But the difference is relatively small. Our simulation suggests that 3.75 may serve as an approximate critical value at the $5 \%$ significance level for all sample sizes used in the bivariate study. This critical value is larger than 3.0 or 3.5 used in univariate outlier detection. See Chen and Liu (1993) and the references therein. For the trivariate case, the difference in the empirical 95-th percentiles of $C_{\max }\left(i, h_{i}^{*}\right)$ is relatively large, indicating that the critical values of $C_{\max }\left(i, h_{i}^{*}\right)$ depend on the dimension of $x_{t}$ and should be adjusted accordingly in practice.

Next, we use simulation to study the power of the proposed joint test statistics. The model used in power study is a bivariate AR(1) model with $\Phi$ given in (20). But the innovational covariance matrix is modified so that the variance of the individual innovation is unity and the correlations between innovations are -0.2. Again, because of the normalization used in defining the test statistics, this change should not have any significant impact on the power study. The sample size used is 200 . For each realization, a single outlier is introduced at the time index $t=100$ with outlier parameter $\boldsymbol{\omega}=$ $(3.5,3.5)^{\prime}$. For each type of outlier, we use the empirical $5 \%$ critical value of Table 1 and tabulate the number of realizations that the corresponding test statistic exceeds the critical value. The power based on 10,000 realizations are $89.1 \%, 96.9 \%, 100 \%$ and $92.1 \%$, respectively for IO, AO, LS and TC. Therefore, for the simple case of an isolated outlier, the proposed joint test statistics have good power in detecting the outlier when the sample size is 200 .

\section{A Detection Procedure}

In practice, the number, location, and type of outliers are unknown a priori, and we use an iterative procedure similar to that of the univariate case to detect outliers. Assuming no outliers at the very beginning, we build a multivariate ARIMA model for the series under study. The model is then used to detect outliers. The primary statistics used to detect outliers are the four joint test statistics $J_{\max }\left(i, h_{i}\right)$ in (19). In the case of multiple significant joint test statistics, we identify the outlier type based on the test that has the smallest empirical $p$-value. For example, if $J_{\max }\left(A, h_{A}\right)$ has the smallest $p$-value at time index $h_{o}$ and the $p$-value is smaller than 0.05 , then we identify an additive outlier at time index $h_{o}$ at the $5 \%$ significance level. When all of the four joint statistics are insignificant at a given level, we use the component statistics $C_{\max }\left(i, h_{i}^{*}\right)$ to check for additional outliers. This step ensures that no component outliers are overlooked. In some cases, the estimated outlier parameter $\hat{\omega}$ may also suggest that the identified outlier only affects some of the components.

Once an outlier is identified, its impact on the underlying time series is removed, using the results 
of Section 3. The adjusted series is treated as a new data set and the detecting procedure is iterated. We terminate the estimation and detection procedure when no significant outliers are detected.

Some remarks on the procedure are in order. First, the proposed procedure detects a single outlier in an iteration. Thus, it may take several iterations to detect multiple outliers. This is a conservative procedure primarily to avoid over-specification of the number of outliers. Second, it could happen that an outlier affects different components differently in a vector time series. This possibility is allowed in the proposed procedure. For instance, a strike can appear as an AO on a production series but as a LS on a sales series if it permanently affects the firm's market share. In this case at the time point of strike we may detect first an AO that affects primarily the first component. We may again identify a LS at the same time point in a subsequent iteration with a significant outlier parameter in the second component. Third, when multiple outliers exist, the proposed procedure may encounter masking or smearing effects of the outliers. This is a complicated problem that is currently under study. Fourth, some refinements of the proposed procedure are possible. For example, under the current procedure an identified outlier is assumed to have effects on all components of a time series and the estimated outlier effect $\hat{\boldsymbol{\omega}}$ is used to remove outlier effects. It might be better to only adjust those components which have a significant $t$-ratio in $\hat{\omega}_{i}$. We don't adopt such a procedure because of simplicity consideration.

\section{Application}

We apply the proposed outlier detection procedure to two real examples. The first example is the well-known gas furnace series of Box, Jenkins and Reinsel (1994) and the second example considers the quarterly series of U.S. initial jobless claims and unemployment rates.

Example 1. Figure 1 shows the time plots of input gas rate in cubic feet per minute, $X_{t}$, and percentage of $\mathrm{CO}_{2}$ in outlet gas, $Y_{t}$, both measured in 9 second time intervals. This series is commonly used in the literature as an example of transfer function models. There are 296 observations. For comparison purposes, we also employ the univariate and transfer function models of Box, Jenkins and Reinsel (1994) for the series. Using the joint estimation and detection procedure of Chen and Liu (1993) and a critical value 3.5 , we obtain the models

$$
\begin{gathered}
\left(1-2.273 B+1.923 B^{2}-0.618 B^{3}\right) X_{t}=-0.002+a_{1 t}, \quad \sigma_{1}=0.129 \\
Y_{t}=53.08+\frac{-0.636 B^{3}-0.264 B^{4}-0.439 B^{5}}{1-0.570 B} X_{t}+\frac{1}{1-1.511 B+0.579 B^{2}} a_{2 t}, \quad \sigma_{2}=0.195
\end{gathered}
$$

where $\sigma_{1}$ and $\sigma_{2}$ are the residual standard error of the input and output series, respectively, after outlier adjustment. The detected outliers are given in Table 3. There are 7 and 6 outliers for model (22) and (23), respectively. If the critical value of outlier detection is set to 3.0 , then there are 17 and 10 outliers, respectively, for the two models. A critical value of 3.5 corresponds to approximately an asymptotic $2.5 \%$ significance level. Note that the two TCs at times 113 and 117 in the input series, that show opposite effects, may suggest a patch of outliers in the period 113-116. Similarly, there maybe a patch of outliers from 265 to 269 in the output series.

Turn to multivariate modeling. Using the chi-square statistic of Tiao and Box (1981) and the Akaike Information Criterion, we employ a bivariate AR(6) model for the series. The first component is the gas rate and the second component is the output $\mathrm{CO}_{2}$ concentration. Applying the proposed detection procedure and using $5 \%$ critical values for the test statistics obtained by interpolation from Tables 1 and 2, we summarize the detection results in Table 4. The critical values are also given in 
the table. Eleven outliers are detected by the procedure. Once an outlier was detected, we removed its effects on the data and re-estimated the bivariate AR(6) model. The estimated outlier parameters $\hat{\omega}=\left(\hat{\omega}_{1}, \hat{\omega}_{2}\right)^{\prime}$ of the 11 outliers are given in Table 5 along with t-ratios of the estimates. Note that the detected TCs at $t=43$ and 55 introduce large $J_{\max }\left(A, h_{A}\right)$ statistics at $t=42$ and 54 . This is understandable because for a vector $\mathrm{AR}(6)$ model, the test statistic $J_{A, h}$ involves filtered values $a_{t}$ for $t=h, h+1, \cdots, h+6$.

It is interesting to compare the detection results between univariate and multivariate methods. First, as shown by Tiao and Box (1981), the marginal models of the bivariate AR(6) model employed are close to those of equations (22) and (23). Therefore, the comparison of detection results can be made fairly. Second, the multivariate method detects fewer outliers than the univariate methods even though the former allows for a larger type-I error. In addition, the outliers detected by the multivariate method are not a subset of those detected by univariate methods. For example, the level shifts at $t=287$ and 288 are not detected by the univariate methods. This demonstrates that multivariate joint detection could be more powerful than univariate methods. Third, the detected IO at $t=265$ clearly highlights the discussion of Subsection 2.1. Specifically, we observe the following:

- The t-ratios of estimated outlier parameters in Table 5 show that the outlier occurred simultaneously to both components.

- As expected, this multivariate IO introduces a patch of outliers in the marginal model of the output series at time indexes 266-269.

- The estimated outlier effect is negative in the input series and the transfer function model shows a negative relationship between the input and output series with a delay of 3 time periods. Consequently, the outlier effects at time indexes 266 and 267 of the output series are positive.

- As expected, the patch of outlier disappears under the multivariate framework.

Fourth, highly significant outliers detected by the univariate methods are also detected by the multivariate method. See outliers at $t=43,55,113$, and 265. Fifth, the classification of outliers is rather consistent between univariate and multivariate methods. Sixth, some minor time differences may occur between univariate and multivariate methods. For example, the univariate outlier at $t=236$ is shown as an outlier at $t=235$ in the multivariate case. Finally, the fact that no outliers occurred in the output series at $t=43$ and 55 where the input series has significant temporary changes suggests that these outlier effects are carried over from the input series to the output series. In practice, this means that the residuals of the output series does not contain additional information of these two outlying observations in the input series.

Example 2. In this example, we consider the U.S. quarterly seasonally adjusted initial jobless claims and unemployment rate from 1948 to 1993 . The initial jobless claims were divided by 100 as in Montgomery et al. (1998). There are 184 observations. Figure 2 shows the time plots of the data. Using the same models as in Montgomery et al. (1998) and the joint estimation-detection procedure of Chen and Liu (1993), we obtain the univariate models

$$
\begin{array}{ll}
(1-0.30 B)\left(1-0.36 B^{4}\right)(1-B) y_{1 t}=\left(1-0.75 B^{4}\right) a_{1 t}, & \sigma_{1}=0.222, \\
(1-0.66 B)\left(1-0.27 B^{4}\right)(1-B) y_{2 t}=\left(1-0.81 B^{4}\right) a_{2 t}, & \sigma_{2}=0.271
\end{array}
$$

where $y_{1 t}$ and $y_{2 t}$ are the initial claims and unemployment rate, respectively. The seasonal parameters in both models are highly significant, even though the data were seasonally adjusted. The detected outliers of the two models are given in Table 6 . There are 4 and 2 outliers for $y_{1 t}$ and $y_{2 t}$, respectively. 
Turn to multivariate detection. We employ a bivariate ARIMA model in the form

$$
\left(I-\Phi_{1} B-\Phi_{2} B^{2}\right)\left(I-\Phi_{4} B^{4}\right) y_{t}=c+\left(I-\Theta_{4} B^{4}\right) \epsilon_{t}
$$

for the data. The detection results are summarized in Table 7 when the $5 \%$ empirical critical values in Table 1 with sample size 200 are used. Only three outliers are detected. The estimated outlier parameters are

$$
[1.249(6.16), 0.334(1.84)],[1.080(4.35), 0.563(2.22)],[0.968(4.14), 0.653(2.62)]
$$

for the outliers at $t=130,108,136$, respectively, where the numbers in parentheses are t-ratios of the estimates. An examination of residual cross-correlation matrices indicates that some minor significant correlations at lag 8. But these serial correlations disappear when the moving-average part is modified to $\left(I-\Theta_{4} B^{4}-\Theta_{8} B^{8}\right) \epsilon_{t}$. The parameter estimates of model (26) before and after outlier adjustment are shown in Table 8 . The three detected outliers have markedly effects on the seasonal parameters and the residual covariance matrix.

Again, it is interesting to compare the detection results of univariate and multivariate models. First, the multivariate model only identifies three outliers whereas the univariate models detect six outliers. In this particular instance, the multivariate outliers form a subset of those identified by univariate methods. Second, the innovational outlier in the unemployment rate at time $t=109$ is caused by the innovational outlier in the initial jobless claims at $t=108$, because there exists no outlier at $t=109$ in the multivariate case. This example clearly demonstrates that (a) an outlier in a component series may be induced by that of another component, and (b) detecting outliers separately for each individual component using a marginal model may result in over-specification of the number of outliers. Third, the significance of the outlier parameters in both components at $t=108$ and 136 indicates that some external disturbances occurred in the U.S. economy at these two time points that affected both the initial jobless claims and unemployment rate. In other words, the impact of these disturbances on the unemployment rate cannot be fully accounted for by that on the initial claims. These two time points were the 4-th quarter of 1974 and 1981, respectively, in which the U.S. economy was in recession as classified by the National Bureau of Economic Research. Thus, the significance of the positive estimates $\hat{\boldsymbol{\omega}}$ at these two periods shows that the economical slowdowns in 1974 and 1981 caused both the initial jobless claims and unemployment rate to rise. In addition, the estimated effect on unemployment rate, $\hat{\omega}_{2}$, represents the additional effect of economical slowdown on unemployment rate beyond that induced by the impact on initial jobless claims. Such information is not evident if one only uses univariate outlier detection. Finally, the significance of both components of $\hat{\boldsymbol{\omega}}$ at $t=108$ and 136 also indicates a possible joint structural break of the series at these two points. Consequently, multivariate outlier detection can be used to study common structural breaks in a vector time series.

\section{REFERENCES}

Box, G.E.P., Jenkins, G.M. and Reinsel, G. (1994) Time Series Analysis: Forecasting and Control, 3rd Ed., New Jersey: Prentice-Hall.

Chang, I., Tiao, G.C. and Chen, C. (1988). Estimation of time series parameters in the presence of outliers. Technometrics, 3, 193-204. 
Chen, C. and Liu, L. (1993). Joint estimation of model parameters and outlier effects in time series. J. Amer. Statist. Assoc., 88, 284-97.

Findley, D.F., Monsell, B.C., Bell, W.R., Otto, M.C., and Chen, B.C. (1998). New capabilities and methods of the X-12-ARIMA seasonal-adjustment program (with discussion). J. Bus. \& Econ. Statist., 16, 127-77.

Fox, A.J. (1972). Outliers in time series. J. Roy. Statist. Soc. Ser. B, 34, 350-63.

Gomez, V. and Maravall, A. (1994a). Program SEATS signal extraction in ARIMA time series: instructions for the user. Working paper ECO 94/28, European University Institute, Florence.

Gomez, V. and Maravall, A. (1994b). Program TRAMO. Time series observations with ARIMA noise, missing observations and outliers: instructions for the user. Working paper ECO 94/31, European University Institute, Florence.

Justel, A., Peña, D. and Tsay, R.S. (1998). Detection of outlier patches in autoregressive time series. Technical Report, Statistics Research Center, Graduate School of Business, University of Chicago.

Li, H. and Tsay, R.S. (1998). A unified approach to identifying multivariate time series models. $J$. Amer. Statist. Assoc., 93, 770-82.

McCulloch, R.E. and Tsay R.S. (1994). Bayesian analysis of autoregressive time series via the Gibbs sampler. J. Time Ser. Analy., 15, 235-50.

Montgomery, A., Zarnowitz, V., Tsay, R.S. and Tiao, G.C. (1998). Forecasting the U.S. unemployment rate. J. Amer. Statist. Assoc., 93, 478-93.

Pankratz, A. (1993). Detecting and treating outliers in dynamic regression models. Biometrika, $80,847-54$.

Tiao, G.C. and Box, G.E.P. (1981). Modeling multiple time series with applications. J. Amer. Statist. Assoc., 76, 802-16.

Tsay, R.S. (1988). Outliers, level shifts, and variance change in time series. J. Forecasting, 7, 1-20. 
Table 1: Empirical quantiles of the $J_{m a x}\left(i, h_{i}\right)$ statistics in (19) based on 10,000 realizations. The models used are in (20) and (21).

\begin{tabular}{|c|c|c|c|c|c|c|}
\hline Sample & Test & \multicolumn{5}{|c|}{ Probability } \\
\hline Size & & $50 \%$ & $90 \%$ & $95 \%$ & $97.5 \%$ & $99 \%$ \\
\hline \multicolumn{7}{|c|}{ (a) Bivariate case $(k=2)$} \\
\hline \multirow[t]{4}{*}{100} & $J_{\max }\left(I, h_{I}\right)$ & 9.74 & 13.03 & 14.35 & 15.60 & 17.34 \\
\hline & $J_{\max }\left(A, h_{A}\right)$ & 9.70 & 13.07 & 14.32 & 15.57 & 16.96 \\
\hline & $J_{\max }\left(L, h_{L}\right)$ & 7.61 & 11.13 & 12.37 & 13.50 & 14.82 \\
\hline & $J_{\max }\left(T, h_{T}\right)$ & 9.58 & 12.95 & 14.27 & 15.43 & 17.05 \\
\hline \multirow[t]{4}{*}{200} & $J_{\max }\left(I, h_{I}\right)$ & 11.20 & 14.66 & 16.01 & 17.47 & 19.06 \\
\hline & $J_{\max }\left(A, h_{A}\right)$ & 11.13 & 14.66 & 15.95 & 17.37 & 19.18 \\
\hline & $J_{\max }\left(L, h_{L}\right)$ & 8.37 & 12.18 & 13.49 & 14.81 & 16.40 \\
\hline & $J_{\max }\left(T, h_{T}\right)$ & 11.04 & 14.55 & 15.87 & 17.19 & 18.67 \\
\hline \multirow[t]{4}{*}{400} & $J_{\max }\left(I, h_{I}\right)$ & 12.60 & 16.19 & 17.63 & 19.06 & 20.81 \\
\hline & $J_{\max }\left(A, h_{A}\right)$ & 12.56 & 16.21 & 17.64 & 18.86 & 20.81 \\
\hline & $J_{\max }\left(L, h_{L}\right)$ & 9.62 & 13.48 & 14.88 & 16.20 & 18.05 \\
\hline & $J_{\max }\left(T, h_{T}\right)$ & 12.57 & 16.13 & 17.53 & 18.96 & 20.83 \\
\hline \multicolumn{7}{|c|}{ (b) Trivariate case $(k=3)$} \\
\hline \multirow[t]{4}{*}{100} & $J_{\max }\left(I, h_{I}\right)$ & 15.55 & 25.00 & 29.56 & 34.23 & 41.43 \\
\hline & $J_{\max }\left(A, h_{A}\right)$ & 15.50 & 25.08 & 29.49 & 34.00 & 41.81 \\
\hline & $J_{\max }\left(L, h_{L}\right)$ & 10.64 & 18.09 & 21.56 & 25.46 & 32.06 \\
\hline & $J_{\max }\left(T, h_{T}\right)$ & 15.48 & 25.14 & 30.05 & 34.81 & 42.18 \\
\hline \multirow[t]{4}{*}{200} & $J_{\max }\left(I, h_{I}\right)$ & 19.20 & 28.45 & 32.10 & 36.72 & 42.10 \\
\hline & $J_{\max }\left(A, h_{A}\right)$ & 19.10 & 28.90 & 33.04 & 37.28 & 43.02 \\
\hline & $J_{\max }\left(L, h_{L}\right)$ & 12.12 & 19.99 & 23.24 & 26.43 & 31.51 \\
\hline & $J_{\max }\left(T, h_{T}\right)$ & 19.12 & 28.86 & 32.85 & 37.18 & 43.29 \\
\hline \multirow[t]{4}{*}{400} & $J_{\max }\left(I, h_{I}\right)$ & 22.86 & 32.24 & 36.12 & 40.04 & 45.09 \\
\hline & $J_{\max }\left(A, h_{A}\right)$ & 22.96 & 32.66 & 36.49 & 40.32 & 45.77 \\
\hline & $J_{\max }\left(L, h_{L}\right)$ & 14.50 & 22.89 & 26.39 & 30.27 & 34.56 \\
\hline & $J_{\max }\left(T, h_{T}\right)$ & 23.10 & 32.79 & 36.67 & 40.67 & 45.47 \\
\hline
\end{tabular}


Table 2: Empirical quantiles of the statistics $C_{\max }\left(i, h_{i}^{*}\right)$ in (19) based on 10,000 realizations. The models used are in (20) and (21).

\begin{tabular}{|c|c|c|c|c|c|c|}
\hline Sample & \multirow[t]{2}{*}{ Test } & \multicolumn{5}{|c|}{ Probability } \\
\hline Size & & \multicolumn{3}{|c|}{$\begin{array}{lll}50 \% & 90 \% & 95 \% \\
\end{array}$} & $97.5 \%$ & $99 \%$ \\
\hline \multicolumn{7}{|c|}{ (a) Bivariate case $(k=2)$} \\
\hline \multirow[t]{4}{*}{100} & $C_{\max }\left(I, h_{I}^{*}\right)$ & 2.89 & 3.39 & 3.58 & 3.74 & 3.96 \\
\hline & $C_{\max }\left(A, h_{A}^{*}\right)$ & 2.89 & 3.39 & 3.57 & 3.73 & 3.94 \\
\hline & $C_{\max }\left(L, h_{L}^{*}\right)$ & 2.61 & 3.18 & 3.35 & 3.52 & 3.71 \\
\hline & $C_{\max }\left(T, h_{T}^{*}\right)$ & 2.87 & 3.37 & 3.55 & 3.74 & 3.95 \\
\hline \multirow[t]{4}{*}{200} & $C_{\max }\left(I, h_{I}^{*}\right)$ & $\overline{3.11}$ & 3.60 & $\overline{3.78}$ & 3.95 & 4.15 \\
\hline & $C_{\max }\left(A, h_{A}^{*}\right)$ & 3.11 & 3.60 & 3.78 & 3.93 & 4.15 \\
\hline & $C_{\max }\left(L, h_{L}^{*}\right)$ & 2.74 & 3.33 & 3.50 & 3.68 & 3.88 \\
\hline & $C_{\max }\left(T, h_{T}^{*}\right)$ & 3.09 & 3.58 & 3.76 & 3.93 & 4.11 \\
\hline \multirow[t]{4}{*}{400} & $C_{\max }\left(I, h_{I}^{*}\right)$ & 3.32 & 3.80 & $\overline{3.96}$ & 4.13 & 4.35 \\
\hline & $C_{\max }\left(A, h_{A}^{*}\right)$ & 3.31 & 3.80 & 3.97 & 4.12 & 4.32 \\
\hline & $C_{\max }\left(L, h_{L}^{*}\right)$ & 2.94 & 3.51 & 3.69 & 3.86 & 4.06 \\
\hline & $C_{\max }\left(T, h_{T}^{*}\right)$ & 3.31 & 3.78 & 3.95 & 4.12 & 4.34 \\
\hline \multicolumn{7}{|c|}{ (b) Trivariate case $(k=3)$} \\
\hline \multirow[t]{4}{*}{100} & $C_{\max }\left(I, h_{I}^{*}\right)$ & 3.01 & 3.48 & 3.64 & 3.79 & 3.96 \\
\hline & $C_{\max }\left(A, h_{A}^{*}\right)$ & 3.24 & 3.93 & 4.18 & 4.44 & 4.74 \\
\hline & $C_{\max }\left(L, h_{L}^{*}\right)$ & 2.77 & 3.54 & 3.83 & 4.11 & 4.52 \\
\hline & $C_{\max }\left(T, h_{T}^{*}\right)$ & 3.23 & 3.94 & 4.18 & 4.45 & 4.78 \\
\hline \multirow[t]{4}{*}{200} & $C_{\max }\left(I, h_{I}^{*}\right)$ & 3.22 & 3.69 & 3.85 & 4.02 & 4.20 \\
\hline & $C_{\max }\left(A, h_{A}^{*}\right)$ & 3.56 & 4.24 & 4.50 & 4.72 & 4.97 \\
\hline & $C_{\max }\left(L, h_{L}^{*}\right)$ & 2.90 & 3.67 & 3.93 & 4.18 & 4.49 \\
\hline & $C_{\max }\left(T, h_{T}^{*}\right)$ & 3.55 & 4.28 & 4.52 & 4.77 & 5.06 \\
\hline \multirow[t]{4}{*}{400} & $C_{\max }\left(I, h_{I}^{*}\right)$ & 3.43 & 3.90 & 4.07 & 4.21 & 4.38 \\
\hline & $C_{\max }\left(A, h_{A}^{*}\right)$ & 3.86 & 4.56 & 4.80 & 5.03 & 5.34 \\
\hline & $C_{\max }\left(L, h_{L}^{*}\right)$ & 3.08 & 3.84 & 4.13 & 4.39 & 4.67 \\
\hline & $C_{\max }\left(T, h_{T}^{*}\right)$ & 3.87 & 4.64 & 4.89 & 5.12 & 5.41 \\
\hline
\end{tabular}


Table 3: Outliers detected for the Gas-Furnace Series using a univariate method with critical value 3.5, where IO, AO, LS, and TC stand for innovational outlier, additive outlier, level shift and temporary change, respectively.

\begin{tabular}{|r|r|r|c|r|r|r|c|}
\hline \multicolumn{4}{|c|}{ (a) Input series: gas rate } & \multicolumn{4}{|c|}{ (b) Transfer function for $\mathrm{CO}_{2}$} \\
\hline Time & Size & t-ratio & Type & Time & Size & t-ratio & Type \\
\hline 43 & 0.770 & 12.20 & TC & 199 & 0.915 & 6.08 & LS \\
55 & -0.718 & -11.38 & TC & 236 & -0.863 & -4.42 & IO \\
91 & 0.286 & 4.53 & TC & 265 & 1.481 & 7.59 & IO \\
113 & -0.479 & -7.59 & TC & 266 & 0.729 & 3.74 & IO \\
117 & 0.248 & 3.92 & TC & 267 & 0.454 & 4.23 & AO \\
198 & -0.534 & -4.15 & IO & 269 & -1.296 & -6.33 & IO \\
262 & 0.607 & 4.72 & IO & & & & \\
\hline
\end{tabular}

Table 4: Results of multivariate outlier detection for the Gas-Furnace Series using a bivariate AR(6) model and $5 \%$ critical values. The number in parentheses for joint tests is the corresponding time index whereas those for component tests are time index and component index.

\begin{tabular}{|r|r|r|r|r|r|r|}
\hline Itera- & \multicolumn{5}{|c|}{ (a) Joint Test Statistics } & \multicolumn{2}{|c|}{ Outlier } \\
\hline tions & $J_{\max }\left(I, h_{I}\right)$ & $J_{\max }\left(A, h_{A}\right)$ & $J_{\max }\left(L, h_{L}\right)$ & $J_{\max }\left(T, h_{T}\right)$ & Time & Type \\
\hline 1 & $39.23(265)$ & $35.70(42)$ & $27.84(199)$ & $41.05(43)$ & 43 & TC \\
2 & $38.54(265)$ & $43.90(54)$ & $26.22(199)$ & $46.15(55)$ & 55 & TC \\
3 & $39.29(265)$ & $27.15(264)$ & $24.46(199)$ & $28.09(264)$ & 265 & IO \\
4 & $16.94(199)$ & $26.27(113)$ & $24.29(199)$ & $26.70(113)$ & 199 & LS \\
5 & $16.01(269)$ & $25.85(113)$ & $16.56(113)$ & $26.24(113)$ & 113 & TC \\
6 & $16.34(262)$ & $16.71(235)$ & $14.49(288)$ & $14.44(261)$ & 288 & LS \\
7 & $16.29(262)$ & $17.56(235)$ & $<14.10$ & $14.55(91)$ & 235 & AO \\
8 & $16.57(269)$ & $<14.10$ & $15.32(287)$ & $14.77(91)$ & 287 & LS \\
9 & $<14.10$ & $14.32(91)$ & $<14.10$ & $15.44(91)$ & & \\
\hline Crit. & 16.82 & 16.80 & 14.19 & 16.70 & & \\
\hline Itera- & \multicolumn{7}{|c|}{ (b) Component Test Statistics } & Outlier \\
\hline tions & $C_{\max }\left(I, h_{I}^{*}\right)$ & $C_{\max }\left(A, h_{A}^{*}\right)$ & $C_{\max }\left(L, h_{L}^{*}\right)$ & $C_{\max }\left(T, h_{T}^{*}\right)$ & Time & Type \\
\hline 9 & $<3.60$ & $3.77(91,1)$ & $<3.60$ & $3.90(91,1)$ & 91 & TC \\
10 & $4.09(262,1)$ & $3.84(197,1)$ & $<3.60$ & $3.88(197,1)$ & 262 & IO \\
11 & $3.74(198,1)$ & $3.92(197,1)$ & $<3.60$ & $3.92(197,1)$ & 197 & TC \\
12 & $<3.60$ & $<3.60$ & $<3.60$ & $<3.60$ & & \\
\hline Crit. & 3.87 & 3.88 & 3.60 & 3.86 & & \\
\hline
\end{tabular}


Table 5: Estimates of outlier parameters for the Gas-Furnace series using a multivariate model, where IO, AO, LS and TC are defined as before in Table 3.

\begin{tabular}{|c|c|r|r|c|c|r|r|}
\hline Time & time & $\hat{\omega}_{1}$ (t-ratio) & $\hat{\omega}_{2}$ (t-ratio) & Time & time & $\hat{\omega}_{1}$ (t-ratio) & $\hat{\omega}_{2}$ (t-ratio) \\
\hline 43 & TC & $0.683(6.41)$ & $-0.019(-0.11)$ & 55 & TC & $-0.613(-6.79)$ & $0.049(0.27)$ \\
265 & IO & $-0.362(-3.40)$ & $1.396(5.86)$ & 199 & LS & $-0.098(-1.51)$ & $.866(4.93)$ \\
113 & TC & $-0.376(-5.12)$ & $-0.067(-0.42)$ & 288 & LS & $0.154(2.04)$ & $0.587(3.23)$ \\
235 & AO & $-0.023(-0.54)$ & $0.437(4.13)$ & 287 & LS & $0.136(1.84)$ & $0.587(3.44)$ \\
\hline 91 & TC & $0.248(3.90)$ & & 262 & IO & $0.559(4.09)$ & \\
197 & TC & $0.149(3.92)$ & & & & & \\
\hline
\end{tabular}

Table 6: Outliers detected for initial jobless claims and unemployment rates using univariate method with critical value 3.5, where IO, AO, LS, and TC stand for innovational outlier, additive outlier, level shift and temporary change, respectively.

\begin{tabular}{|r|r|r|c|r|r|r|c|}
\hline \multicolumn{4}{|c|}{ (a) Initial jobless claims } & \multicolumn{4}{|c|}{ (b) Unemployment rates } \\
\hline Time & Size & t-ratio & Type & Time & Size & t-ratio & Type \\
\hline 108 & 1.137 & 5.13 & IO & 109 & 1.097 & 4.04 & IO \\
130 & 1.493 & 9.08 & TC & 140 & 0.417 & 4.30 & AO \\
136 & 0.999 & 5.43 & LS & & & & \\
141 & -0.971 & -5.25 & LS & & & & \\
\hline
\end{tabular}

Table 7: Results of multivariate outlier detection for the initial jobless claim and unemployment series using a bivariate seasonal ARIMA model and $5 \%$ critical values. The number in parentheses for joint tests is the corresponding time index whereas those for component tests are time index and component index.

\begin{tabular}{|r|r|r|r|r|r|r|}
\hline Itera- & \multicolumn{3}{|c|}{ (a) Joint Test Statistics } & \multicolumn{2}{|c|}{ Outlier } \\
\hline tions & $J_{\max }\left(I, h_{I}\right)$ & $J_{\max }\left(A, h_{A}\right)$ & $J_{\max }\left(L, h_{L}\right)$ & $J_{\max }\left(T, h_{T}\right)$ & Time & Type \\
\hline 1 & $31.66(130)$ & $36.45(130)$ & $<13.50$ & $40.33(130)$ & 130 & TC \\
2 & $20.08(108)$ & $<13.50$ & $<13.50$ & $16.65(141)$ & 108 & IO \\
3 & $17.25(136)$ & $<13.50$ & $<13.50$ & $<13.50$ & 136 & IO \\
4 & $15.53(25)$ & $<13.50$ & $<13.50$ & $14.05(141)$ & $:$ & \\
\hline Crit. & 16.01 & \multicolumn{3}{|c|}{ (b) Component Test Statistics } & \multicolumn{2}{c|}{ Outlier } \\
\hline Itera- & \multicolumn{7}{|c|}{15.87} & & \\
\hline tions & $C_{\max }\left(I, h_{I}^{*}\right)$ & $C_{\max }\left(A, h_{A}^{*}\right)$ & $C_{\max }\left(L, h_{L}^{*}\right)$ & $C_{\max }\left(T, h_{T}^{*}\right)$ & Time & Type \\
\hline 4 & $3.59(25,2)$ & $<3.50$ & $<3.50$ & $3.61(141,1)$ & & \\
\hline Crit. & 3.78 & 3.78 & 3.50 & 3.76 & & \\
\hline
\end{tabular}


Table 8: Parameter estimates of model (26) before and after multivariate outlier detection. The values in parentheses are standard errors.

\begin{tabular}{|c|rr|r|r|rr|r|r|r|r|r|}
\hline $\boldsymbol{c}^{\prime}$ & \multicolumn{1}{|c|}{$\Phi_{1}$} & \multicolumn{2}{c|}{$\Phi_{2}$} & \multicolumn{2}{|c|}{$\Phi_{4}$} & \multicolumn{1}{|c|}{$\Theta_{4}$} & \multicolumn{1}{c|}{$\boldsymbol{\Sigma}$} \\
\hline \multicolumn{10}{|c|}{ (a) Before outlier adjustment } \\
\hline .139 & 1.31 & -.17 & -.25 & .11 & .13 & -.19 & .02 & .17 & .081 & .056 \\
$(.089)$ & $(.10)$ & $(.09)$ & $(.11)$ & $(.07)$ & $(.18)$ & $(.08)$ & $(.22)$ & $(.15)$ & & \\
.054 & .59 & 1.16 & -.31 & -.36 & .06 & -.08 & -.07 & -.09 & .056 & .073 \\
$(.086)$ & $(.09)$ & $(.09)$ & $(.11)$ & $(.09)$ & $(.13)$ & $(.21)$ & $(.17)$ & $(.23)$ & & \\
\hline \multicolumn{10}{|c|}{ (b) After outlier adjustment } \\
\hline .322 & 1.37 & -.22 & -.33 & .15 & -.21 & -.12 & -.55 & .18 & .049 & .036 \\
$(.13)$ & $(.09)$ & $(.07)$ & $(.11)$ & $(.06)$ & $(.16)$ & $(.07)$ & $(.17)$ & $(.12)$ & & \\
.142 & .66 & 1.12 & -.35 & -.33 & -.06 & -.28 & -.22 & -.13 & .036 & .060 \\
$(.13)$ & $(.10)$ & $(.08)$ & $(.12)$ & $(.08)$ & $(.15)$ & $(.17)$ & $(.18)$ & $(.20)$ & & \\
\hline
\end{tabular}



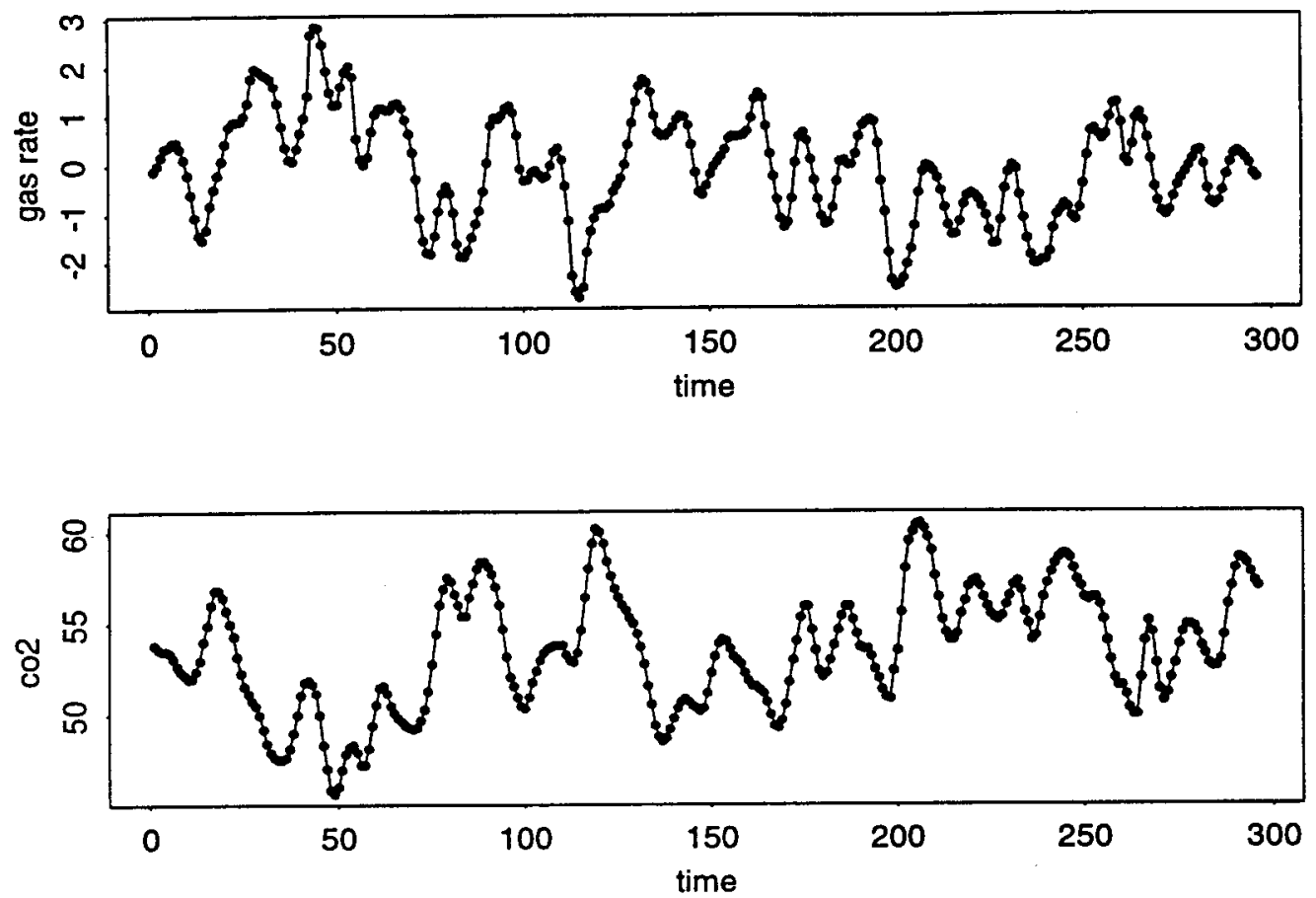

Figure 1: Time plots of Input Gas Rate and Percentage of $\mathrm{CO}_{2}$ in Output 

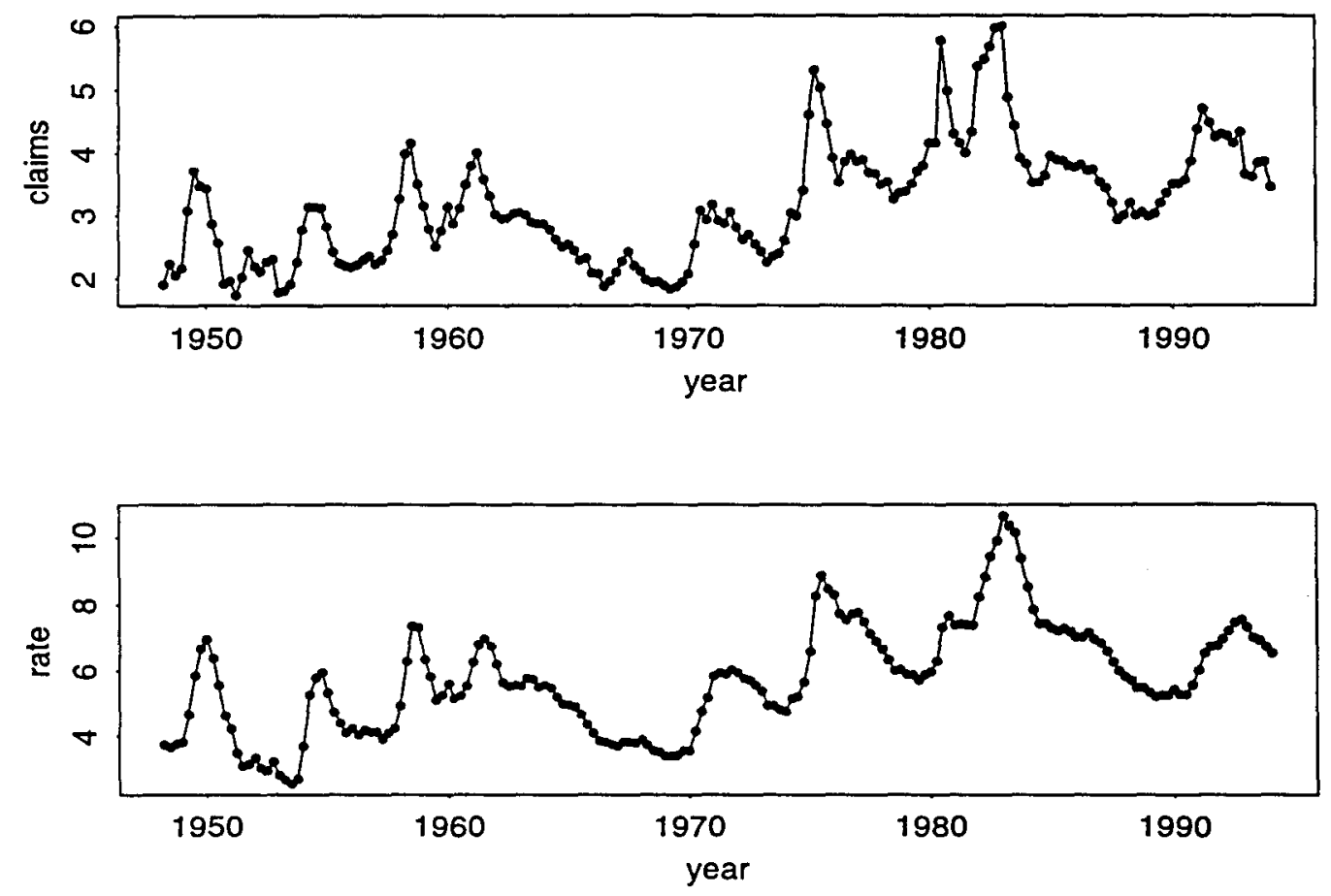

Figure 2: Time plots of U.S. quarterly initial jobless claims (divided by 100) and unemployment rate: 1948-1993. The data were seasonally adjusted. 